\title{
Phenylalanine Alters the Mean Power Frequency of Electroencephalograms and Plasma L-DOPA in Treated Patients with Phenylketonuria
}

\author{
WILMA KRAUSE, CHARLES EPSTEIN, ALLEN AVERBOOK, PHILIP DEMBURE, AND \\ LOUIS ELSAS \\ Division of Medical Genetics, Departments of Pediatrics and Neurology, Emory University, \\ Atlanta, Georgia 30322
}

\begin{abstract}
Phenylketonuria is a human model for the study of the effects of phenylalanine on brain function. We found previously a correlation between high blood phenylalanine, prolonged performance times on neuropsychological tests of higher integrative function, and decreased urinary dopamine in 10 patients. In this protocol we examine changes in triplicate of plasma dihydroxyphenylalanine (L-DOPA) and the mean power frequency of the electroencephalogram in eight additional older patients with phenylketonuria using longer intervals in a blinded, cross-over design. Mean power frequency was obtained by Fourier transform of the power spectrum from traditional eight channel electroencephalograms. Plasma $\mathrm{L}-\mathrm{DOPA}$ was quantitated by radioenzymatic methods. In all patients statistically significant decreases were found in the mean power frequency of the electroencephalogram and in plasma $L$-DOPA when plasma $L$-phenylalanine increased. These findings were reversible and correlated in the reverse direction when plasma $L$-phenylalanine was reduced. Thus changes in the mean power frequency of electroencephalograms and circulating L-DOPA offer sensitive parameters of human brain function in vivo. These findings indicate reversible effects of elevated plasma phenylalanine on electrical function of the brain which may be mediated in part through inhibition of catecholamine synthesis. $(\mathrm{Pe}$ diatr Res 20:1112-1116, 1986)
\end{abstract}

\section{Abbreviations}

PKU, phenylketonuria

MPF, mean power frequency

L-DOPA, dihydroxyphenylalanine

PKU is the prototypic experiment of nature by which adverse effects of high circulating blood phenylalanine on human brain structure and function are studied. Two different pathological phenomena are postulated as caused by elevated concentrations of phenylalanine. First, in the developing human brain, from fetal life to 6 months of age, irreversible changes in myelin, brain structure, and neuronal migration are described (1-5). Second, elevated blood phenylalanine concentrations may impair the fully myelinated human brain by reversible mechanisms involving neurotransmitter production (6-9). This latter phenomenon

Received April 2. 1986; accepted June 6, 1986

Address all correspondence and request for reprints to Dr. Louis J. Elsas, Medical Genetics, Emory University, 2040 Ridgewood Drive, Atlanta, GA 30322

This work was supported by Grant 12-110 from the March of Dimes and a Grant 2-M01-RR00039-25 from the National Institutes of Health. has considerable importance when one considers the adverse effects of diet discontinuation on intellectual achievement and behavior of early treated, 6 yr olds with PKU (10).

Recently, we approached the issue of developing noninvasive, sensitive discriminants of brain function by quantitating catecholamine production and neuropsychological tests of performance in patients with high and low blood phenylalanine concentrations. In this study we found prolonged performance times in tests of higher integrative function but not for tests of lower integrative function when plasma phenylalanine rose (8). When plasma phenylalanine concentration was elevated, urinary excretion of dopamine decreased (8). Since in well patients it is not ethical to measure neurotransmitters in brain or cerebrospinal fluid and since neuropsychological tests are subject to many variables such as the individual's competence, environmental conditions, and rapport with examiners, we sought better tests which were safe, noninvasive, repeatable, and subject to statistical analysis. We considered the use of electrophysiological measurements. Rolle-Daya studied the standard EEG in patients with PKU and reviewed earlier studies (11). Most of these observations described prevention of nonspecific EEG changes, such as paroxysmal discharges, if newborns with phenylalanine hydroxylase deficiency were treated by diet (11). Using computerized analytical techniques, Donker et al. (12) investigated the MPF in EEGs during oral phenylalanine loading with 100 to $150 \mathrm{mg} /$ $\mathrm{kg}$ per day in six older patients who varied in age of initiation of treatment and in mental achievement. They observed low frequency, $\delta$ band activity, and a change in frequency of dominant rhythms with increased blood phenylalanine. No irritative or spike and wave complexes were seen (12). Di Giorgis et al. (13) in their prospective study of PKU infants in the 1st yr of life concluded that the longer and higher the increase was in blood phenylalanine concentration, the more prone the infant was to epileptiform alterations of EEG. None of the groups investigated older, early-treated patients under conditions in which electrical changes were used to monitor brain function within the same patient. We chose to quantitate the effects of changes in plasma phenylalanine on the EEG, using computerized electroencephalography.

In this study we test the hypothesis that increased plasma and consequent intracerebral phenylalanine concentrations compete with tyrosine for conversion to L-DOPA through tyrosine hydroxylase. This competitive inhibition was previously demonstrated by Udenfriend (14) who described a $\mathrm{K}_{\mathrm{i}}$ of $1.7 \times 10^{-5} \mathrm{M}$ for phenylalanine on tyrosine hydroxylase in vitro. The product of this reaction, L-DOPA, occurs in $\mathrm{ng} / \mathrm{ml}$ of plasma and of cerebrospinal fluid. It can be specifically quantitated by modifying a radioenzymatic assay (15). We tested our hypothesis by simultaneously quantitating changes in the mean power frequency of traditional eight-channel electroencephalograms 
through Fourier transformations and by measuring plasma $\mathrm{L}$ DOPA. This clinical-research protocol involved older, treated patients with phenylketonuria in a double-blinded, cross-over design. During each study interval their plasma phenylalanine concentrations were uniquely altered and maintained at high or low concentrations for periods sufficient to enable triplicate measurements of L-DOPA, MPF, and L-phenylalanine during the last 3 days of each condition.

\section{METHODS}

Clinical design. Parents and children were informed of the research project and gave their consent for study. Eight, early treated (before 1 month of age) patients with classic mutations in phenylalanine hydroxylase were aged 7 to $10 \mathrm{yr}$ at the time they were admitted to the Emory University Clinical Research Facility. The study design utilized three, consecutive, 9-day periods during which time the patients, parents, and the laboratory personnel were not aware of the dietary phenylalanine content. Measurements were made in triplicate on the last 3 days of each study period. No patient had a history of seizures. Seven patients were on a low-high-low protocol, i.e. during the first and third periods, phenylalanine was restricted in the patients' diets to concentrations previously used to reduce plasma phenylalanine. During the second period, dietary L-phenylalanine was raised to achieve levels 10 to 15 times normal. The total diet was standardized and unchanged during the 27 days save for the addition of tasteless L-phenylalanine to the formula during the loading phase. One patient (D.R.) was on a high-low-high protocol. He had been on diet for the 1st yr of life, was lost to followup and off diet for $10 \mathrm{yr}$.

A profile of these patients is presented in Table 1. N.G. and D.R. who scored low on their performance tests had significant histories of poor dietary compliance after 1 yr of age.

Biochemical analyses. Blood was drawn in the semifasting state (late morning) for quantitation of plasma amino acids and L-DOPA on days 7,8 , and 9 of each study period to standardize for diurnal variation in the ratios of phenylalanine and tyrosine (16). Amino acids were analyzed by ion exchange chromatography on a Beckman Model $118 \mathrm{CL}$ or 6300 using lithium buffers (Beckman Instruments, Inc., Palo Alto, CA). L-DOPA was analyzed using a modification of the single-isotope, radioenzymatic assay for catecholamines in which samples are run with and without DOPA decarboxylase (to convert DOPA to dopamine). The difference in dopamine concentrations between the two assays measures DOPA (15).

Electroencephalography and statistical analysis. Eight channel EEGs were recorded with eyes open in the relaxed waking state as previously described (17). Channel one was left, fronto-frontal; channel two was left, centro-temporal; channel three was left, parieto-temporal; and channel seven left, centro-occipital. Channels four, five, six, and eight were the counterparts on the right. All studies were performed in the late afternoon. Ten, 2-s, artifact-free samples were analyzed daily by fast Fourier trans- form for each subject on the last 3 days of each of the three study periods (18). To assess differences in MPF between study periods, the average of the three MPF values for 3 days of each period were compared. Results for each channel for all seven subjects on the low-high-low protocol were arrayed by condition (17). Variation between the average MPFs was analyzed by two-way analysis of variance for each channel for all seven subjects using the ABSTAT package (Anderson-Bell, Denver, CO). The LDOPA results were similarly arrayed and the same statistical methods were used to analyze between-condition variation.

\section{RESULTS}

Results of electroencephalographic analyses in all eight patients are depicted in Figure 1. Each bar height represents the mean of three MPF results for the 3 successive days at the end of each diet condition bracketed by $1 \mathrm{SD}$. In the first seven patients on a low-high-low dietary phenylalanine protocol, the bar for condition two is the shortest, indicating slowing in MPF during high plasma phenylalanine concentrations. This pattern is reversed in the eighth patient (D.R.) who was on a high-low-high dietary protocol and in whom the middle bar (second condition) was highest. The reversibility of EEG slowing is readily seen in the first seven patients by comparing the bar for condition one and three (lower plasma phenylalanine) with condition two (higher plasma phenylalanine). Again, the reverse holds for the eighth patient, D.R.

The relationships of plasma phenylalanine and L-DOPA concentrations to the MPF of channel seven are arrayed in Table 2. Interperiod differences in plasma phenylalanine concentrations varied from $402 \mu \mathrm{mol}$ to $1358 \mu \mathrm{mol} /$ liter $(\mu \mathrm{M})$. The highest plasma phenylalanine in any subject was $1877 \mu \mathrm{M}(31 \mathrm{mg} / 100$ $\mathrm{ml}$ ). Slowing in MPF occurred in all subjects during periods of elevated plasma phenylalanine concentrations and the effect was reversible when dietary phenylalanine was lowered. The difference in MPF (slowing) between control phenylalanine concentration and elevated phenylalanine concentration was significant in all channels using two-way analyses of variance $(p<0.01)$. The most consistent difference among conditions was seen in channel seven (left centro-occipital).

The relationships of changes in plasma phenylalanine concentrations to changes in the EEG mean power frequency for all eight patients are summarized in Figure 2. Each patient had two "changes" from dietary conditions of high to low or low to high phenylalanine. Each point in quadrant I indicates an increase in MPF with lowering of plasma phenylalanine and each point in quadrant III indicates the reverse. All changes fit into these two quadrants, i.e. a decrease in MPF was associated with an increase in plasma phenylalanine and vice versa.

Consideration was then given regarding whether these changes in brain wave activity fell within normal or abnormal ranges. The solid line in Figure 3 depicts the mean of triplicate observations for the average mean power frequency among 19 normal children aged 5 to $14 \mathrm{yr}$. The broken line is the $95 \%$ prediction

Table 1. Identification of patients by age, IQ, and achievement*

\begin{tabular}{|c|c|c|c|c|c|c|}
\hline \multirow[b]{2}{*}{ Patient } & \multirow[b]{2}{*}{ Age (yr) } & \multirow[b]{2}{*}{ Full scale IQ } & \multirow[b]{2}{*}{ Verbal/performance IQ } & \multicolumn{3}{|c|}{ Wide range achievement test } \\
\hline & & & & Reading & Spelling & Arithmetic \\
\hline C.D. & $84 / 12$ & 96 & $97 / 95$ & 99 & 97 & 91 \\
\hline J.C. & $107 / 12$ & 81 & $73 / 92$ & 85 & 85 & 83 \\
\hline J.J. & $86 / 12$ & 87 & $96 / 80$ & 100 & 100 & 92 \\
\hline M.T. & $113 / 12$ & 118 & $120 / 109$ & 117 & 114 & 108 \\
\hline M.Y. & $71 / 12$ & 99 & $96 / 102$ & 99 & 100 & 78 \\
\hline N.G. & $102 / 12$ & 59 & $59 / 65$ & 84 & 79 & 65 \\
\hline S.H. & $99 / 12$ & 107 & $108 / 105$ & 97 & 90 & 92 \\
\hline D.R. & $117 / 12$ & 79 & $85 / 75$ & 96 & 87 & 71 \\
\hline
\end{tabular}

* The Wechsler Intelligence Scale for children (revised) was used to determine IQ. Scores given for the wide range achievement test are standard scores. 

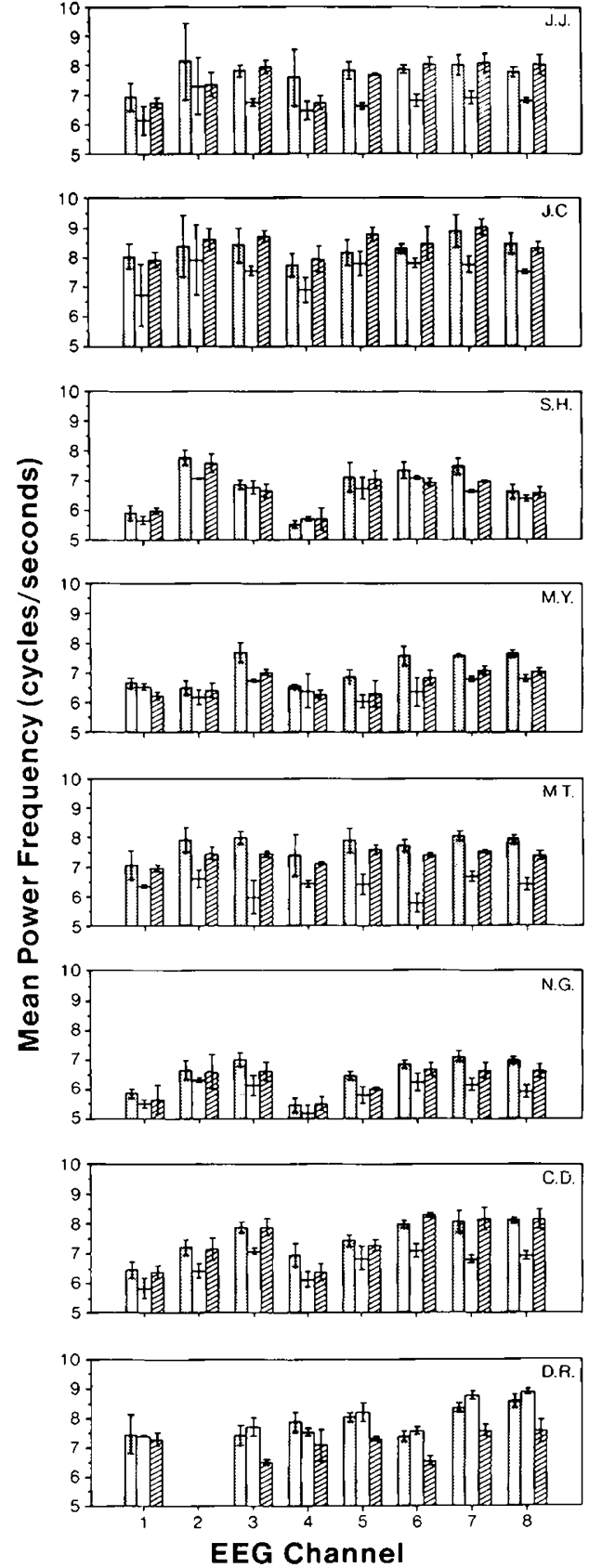

Fig. 1. The average MPF for each of eight channels for all three diet conditions for all patients. Each bar represents the average of three values for 3 consecutive days on each diet and condition bracketed by 2 SDs. Conditions 1, 2, and 3 are indicated by gray, open, and hatched bars, respectively. These conditions were indicative of the low-high-low dietary phenylalanine for the first seven patients (J.J., J.C., S.H., M.Y., M.T., N.G., and C.D.) and high-low-high protocol for the eighth patient, D.R. (see Table 2).

limits for this mean, analyzed using a linear statistical model, which included variance in both control and study groups (19). Superimposed are the mean changes in mean power frequency when low dietary phenylalanine (open circle) was increased to high phenylalanine intake (closed circles). Channel seven was chosen because of low variability on replicate observations for both control and study patients. The data indicate that all of the children with PKU had a MPF which fell within the normal range. Five of the eight had lowered MPF which fell within the $95 \%$ prediction limits while three had slowed EEGs which fell completely outside this normal range when plasma phenylalanine rose (Fig. 3).

Plasma L-DOPA concentrations also decreased in most of the patients when blood phenylalanine increased. Conversely, when blood phenylalanine fell, L-DOPA concentrations rose. Results of all eight patients are depicted in Figure 4. Each point represents the change in L-DOPA which occurred with the change in plasma phenylalanine concentration. Points in quadrant I indicate an increase in L-DOPA with decrease in plasma phenylalanine and quadrant III shows the reverse. A plot of these changes demonstrates that with two exceptions all changes fell into these quadrants. These two exceptions occurred when primary effects were seen but reversibility lagged.

Linear regression analysis was performed using changes in phenylalanine as the independent variable and changes in $\mathrm{L}-$ DOPA and MPF as dependent variables. A multiple correlation coefficient of $0.8172 \pm 0.1995$ was found for changes in $\mathrm{L}$ phenylalanine and L-DOPA. F test analysis gave a value of 28.137 which was significant at $p<0.01$. The regression coefficient was -0.00027288 which means that for a $1 \mathrm{mM}$ increase in plasma L-phenylalanine concentration, an average decrease of $0.3 \mathrm{ng} / \mathrm{ml}$ plasma L-DOPA was observed. A similar statistically significant relationship was found between the changes in plasma L-phenylalanine and changes in MPF. The multiple correlation coefficient was $0.9439 \pm 0.3452$ with an $F$ value of 114.430 for the analysis of variance for the regression $(p<0.01)$. The regression coefficient was -0.00095218 which indicated that for every increase in plasma phenylalanine concentration of $1 \mathrm{mM}$, there was an average decrease of 0.95 CPS in the MPF of the EEG.

After breaking the research protocol, overt behavioral symptoms were noted in some patients when blood phenylalanine was elevated. One patient, N.G., complained of headache, and one patient, M.T., became less interested in activity and more in taking naps, an uncharacteristic behavior. From previously completed assessment tools, parents and medical personnel noted loss of attentiveness and increase in mood swings when blood phenylalanine was high, plasma L-DOPA low, and MPF of the EEG slowed.

\section{DISCUSSION}

Several investigations of the relationship between the EEG and PKU have been published during the past 30 yr. Fois et al. (20) in 1955 reported seizures in their PKU patients. Improvement was noted after dietary treatment (20). In 1966 Clayton et al. (21) addressed the effect of acute phenylalanine loading on the EEG in children who had begun treatment for phenylketonuria at varying ages ( 16 days to nearly $9 \mathrm{yr}$ ). They recorded the EEG for $1 \mathrm{~h}$ after the loading dose and intermittently for 3 to $5 \mathrm{~h}$ thereafter. Changes in the EEG were seen in seven of the 12 children, but they neither sought nor demonstrated a correlation between the degree of increase in blood phenylalanine concentration and the presence or absence of EEG changes (21).

Rolle-Daya et al. (11) reviewed 90 patients in 1975. Seventythree percent of the patients who were treated early (before 3 months of age) had normal EEGs, 23\% had mild background abnormalities and $4 \%$ had paroxysmal discharges. By contrast only $31 \%$ of the patients diagnosed after 6 months of age had normal EEGs. In the early treated group for whom data were available, an increase in background abnormalities was noted in three of 14 subjects after discontinuation of the therapeutic diet. These abnormalities were categorized as bursts of high-voltage $\delta$ rhythms during waking (11).

This early work prompted Donker et al. (12) to apply the technique of computer analysis of the EEG to detect more subtle changes during acute loading of patients with phenylketonuria. They saw primarily occurrences of activity in the low frequency band and change of frequency in dominant rhythms (12). These observations suggested to us that the mean frequency could be a 
Table 2. Comparison of plasma phenylalanine, plasma L-DOPA, and EEG*

\begin{tabular}{|c|c|c|c|c|c|}
\hline Patient & Condition & $\begin{array}{c}\text { Dietary phenylalanine } \\
(\mathrm{mg} / \mathrm{kg} / \text { day })\end{array}$ & $\begin{array}{c}\text { Plasma phenylalanine } \\
(\mu \mathrm{M})\end{array}$ & $\begin{array}{c}\text { Plasma L-DOPA } \\
(\mathrm{ng} / \mathrm{ml})\end{array}$ & $\begin{array}{l}\text { MPF channel } 7 \\
\text { (c/s) }\end{array}$ \\
\hline \multirow{3}{*}{ C.D. } & 1 & 13 & $861 \pm 155 \dagger$ & $1.37 \pm 0.341 \dagger$ & $8.103 \pm 0.370 \dagger$ \\
\hline & 2 & 101 & $1675 \pm 10$ & $0.96 \pm 0.068$ & $6.823 \pm 0.123$ \\
\hline & 3 & 13 & $799 \pm 181$ & $0.99 \pm 0.097$ & $8.193 \pm 0.396$ \\
\hline \multirow[t]{3}{*}{ J.C. } & 1 & 13 & $582 \pm 233$ & $0.81 \pm 0.16$ & $8.898 \pm 0.544$ \\
\hline & 2 & 92 & $1697 \pm 171$ & $0.44 \pm 0.18$ & $7.774 \pm 0.292$ \\
\hline & 3 & 13 & $339 \pm 82$ & $0.85 \pm 0.27$ & $9.021 \pm 0.268$ \\
\hline \multirow[t]{3}{*}{ J.J. } & 1 & 14 & $671 \pm 130$ & $1.10 \pm 0.17$ & $8.044 \pm 0.334$ \\
\hline & 2 & 118 & $1877 \pm 130$ & $0.73 \pm 0.22$ & $6.930 \pm 0.226$ \\
\hline & 3 & 15 & $661 \pm 101$ & $1.26 \pm 0.16$ & $8.111 \pm 0.323$ \\
\hline \multirow[t]{3}{*}{ M.T. } & 1 & 12 & $545 \pm 174$ & $0.71 \pm 0.047$ & $8.027 \pm 0.163$ \\
\hline & 2 & 99 & $1462 \pm 30$ & $0.66 \pm 0.136$ & $6.659 \pm 1.77$ \\
\hline & 3 & 12 & $940 \pm 99$ & $0.73 \pm 0.101$ & $7.503 \pm 0.062$ \\
\hline \multirow[t]{3}{*}{ M.Y. } & 1 & 14 & $41 \pm 31$ & $2.31 \pm 0.12$ & $7.599 \pm 0.036$ \\
\hline & 2 & 95 & $1217 \pm 276$ & $1.60 \pm 0.18$ & $6.789 \pm 0.095$ \\
\hline & 3 & 13 & $815 \pm 163$ & $1.52 \pm 0.09$ & $7.070 \pm 0.160$ \\
\hline \multirow[t]{3}{*}{ N.G. } & 1 & 15 & $649 \pm 163$ & $0.43 \pm 0.107$ & $7.084 \pm 0.206$ \\
\hline & 2 & 70 & $1353 \pm 80$ & $0.40 \pm 0.035$ & $6.148 \pm 0.221$ \\
\hline & 3 & 13 & $936 \pm 115$ & $0.52 \pm 0.095$ & $6.617 \pm 0.278$ \\
\hline \multirow[t]{3}{*}{ S.H. } & 1 & 14 & $32 \pm 17$ & $1.09 \pm 0.14$ & $7.491 \pm 0.260$ \\
\hline & 2 & 96 & $1172 \pm 41$ & $0.78 \pm 0.09$ & $6.620 \pm 0.060$ \\
\hline & 3 & 14 & $94 \pm 10$ & $1.11 \pm 0.14$ & $6.948 \pm 0.055$ \\
\hline \multirow[t]{3}{*}{ D.R. } & 1 & 95 & $1054 \pm 72$ & $0.53 \pm 0.050$ & $8.314 \pm 0.150$ \\
\hline & 2 & 9 & $329 \pm 50$ & $0.45 \pm 0.038$ & $8.700 \pm 0.135$ \\
\hline & 3 & 95 & $1535 \pm 282$ & $0.41 \pm 0.161$ & $7.555 \pm 0.204$ \\
\hline
\end{tabular}

* Analysis of variance of plasma phenylalanine, plasma L-DOPA, and EEG/MPF (channel 7) for condition 1 and 2 showed a significant difference in all seven patients on the low-high-low protocol $(p \leq 0.01)$. Similar analysis of variance of values for conditions 2 and 3 also demonstrated a significant difference $(p \leq 0.01)$.

$\dagger$ Numbers indicate mean \pm 1 SD of triplicate observations.

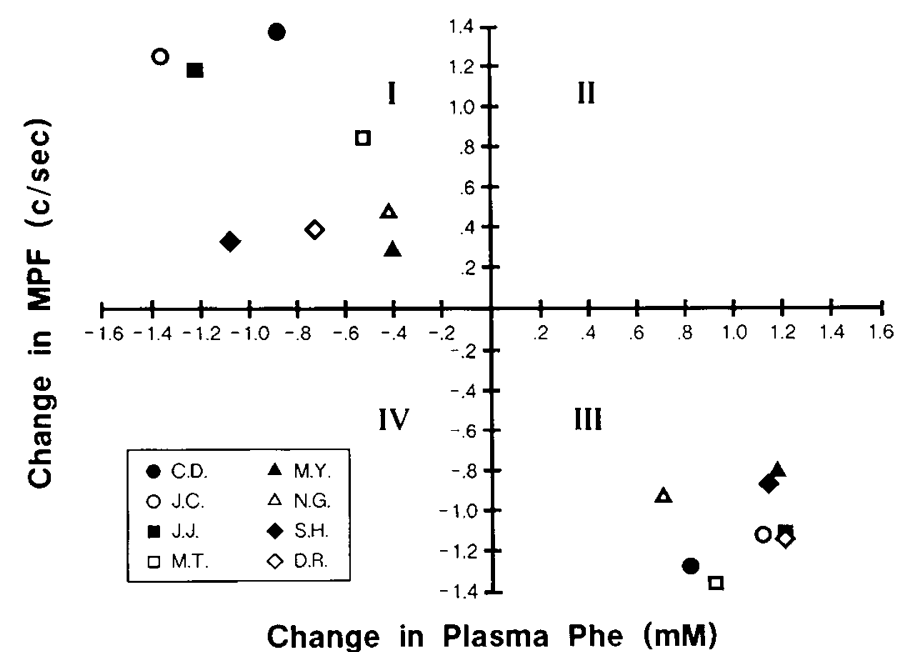

Fig. 2. The relationship of changes in plasma phenylalanine to changes in MPF in channel 7 during dietary manipulation of phenylalanine. Each patient is represented by a symbol that appears twice on the graph, indicating the difference in MPF between the first and second and the second and third condition plotted against parallel changes in plasma phenylalanine.

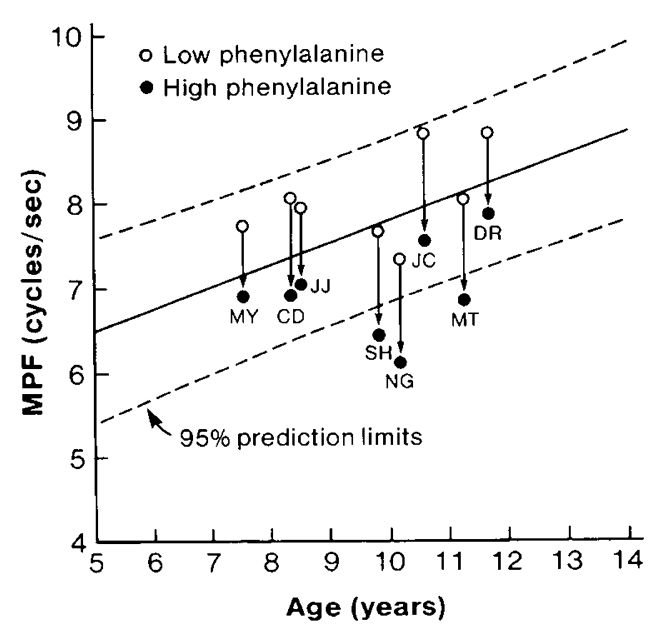

Fig. 3. Normal, age-related limits of the MPF and the effect of diet in phenylketonuria. The MPF for channel 7 was obtained in triplicate for 19 normal children, analyzed using a linear statistical model, and displayed as the mean (solid line) bounded by its $95 \%$ prediction limits (broken line). Data are superimposed for each of the eight patients with PKU comparing their MPF on low and high phenylalanine diets. 


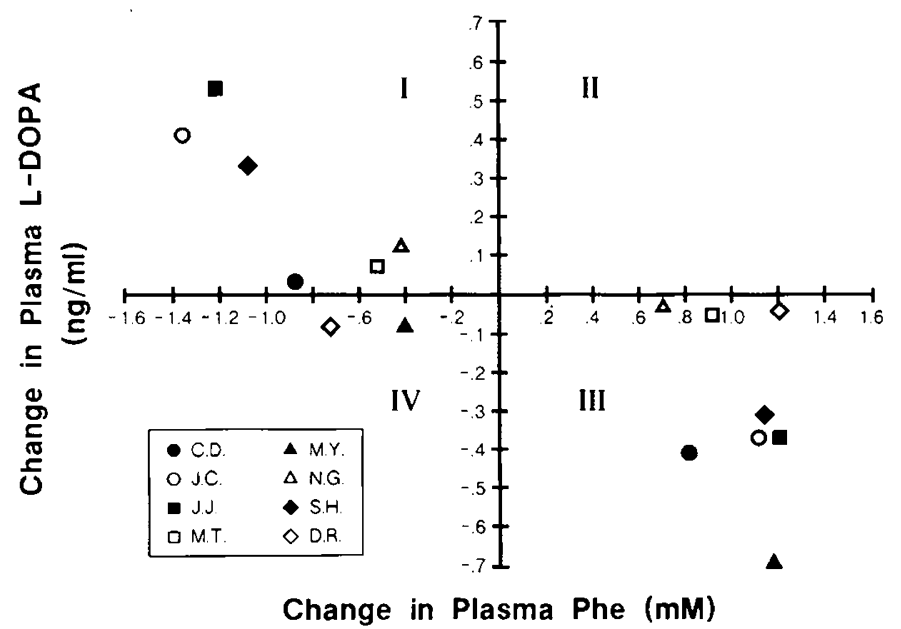

Fig. 4. The relationship of changes in plasma phenylalanine to changes in plasma L-DOPA during dietary manipulation of phenylalanine. Each patient is represented by a symbol that appears twice on the graph, indicating the difference in plasma L-DOPA between the first and second and the second and third condition plotted against parallel changes in plasma phenylalanine.

sensitive parameter with which to follow chronic changes in blood phenylalanine on brain function. As shown in Figure 1 and Table 2 our patients showed consistent slowing of EEGMPF during moderate phenylalanine elevations with reversal of the effect on lowering plasma phenylalanine concentrations. Lowering of the MPF indicates an increase in power concentration in the $\delta$ or slow wave frequencies which is indicative of decreased alertness. These are not specific effects of phenylalanine, since similar changes are seen in toxic encephalopathies such as those produced by renal failure, Reye's syndrome, and central nervous system depressants. Nor are they necessarily accompanied by clinical manifestations of encephalopathy (22, 23). The assessment of MPF during relaxed wakefulness is important since sleep may obscure changes induced by changes in blood phenylalanine (24).

There is agreement in the neuropharmacology literature that catecholamines modulate wakefulness (9) and that precursor nutrients alter neurotransmitter production (25-28). Tyrosine hydroxylase resides primarily in the brain and adrenal medulla. It has been identified in other organs but is associated with synaptic vesicles, is intraneuronal, and disappears on denervation (29). Its product, L-DOPA, is a neutral amino acid with a $\mathrm{ng} / \mathrm{ml}$ concentration in both plasma and cerebrospinal fluid which suggests that it is in equilibrium with the central nervous system. Therefore, changes in plasma L-DOPA concentration probably reflected variation in brain concentrations (29). Pardridge (25) has demonstrated a 2- to 4-fold concentration gradient across the blood-brain barrier for phenylalanine. With blood phenylalanine in the $10^{-4} \mathrm{M}$ range it is probable that intracerebral phenylalanine inhibited tyrosine hydroxylase and reduced LDOPA production by competitive and therefore reversible mechanisms.

These changes of blood L-phenylalanine concentration, LDOPA production and electrical activity of the brain, reflect cognitive function and the ability to concentrate. Our earlier studies used choice-reaction times and found a slowing in higher integrative response time (8). However, to confirm these observations, a more extensive battery of tests of cognitive function is needed which are not subject to learning artifacts, are valid regarding organic brain disease, and provide statistically quantifiable changes within the same individual. Such tests of neuropsychological performance, combined with changes in plasma LDOPA and the mean power frequency of the EEG, would make good clinical parameters in deciding on diet discontinuation for older patients with PKU.

Acknowledgments. The authors express their appreciation to Dr. Bahjat Faraj and Mr. Vernon Camp of the Department of Radiology for performing the catecholamine assays, to Ms. Jean Drumheller for the plasma amino acid analyses, to Dr. Elmer C. Hall and Mr. Edward Strumlauf for statistical advice, and to Ms. Marcia Sternberg for typing this manuscript.

\section{REFERENCES}

1. Agrawal HC, Bone AH, Davison AN 1970 Effects of phenylalanine on protein synthesis in the developing rat brain. Biochem $J$ 117:325-331

2. Johnson RC, Shah SN 1980 Effects of alpha-methylphenylalanine plus phenylalanine treatment during development of myelin in rat brain. Neurochem Res 5:709-718

3. Crome L, Pare CMB 1960 Phenylketonuria: a review and a report of the pathological findings in four cases. J Ment Sci 106:862-883

4. Agrawal HC, Davison AN 1973 Myelination and amino acid imbalance in the developing brain. In: Himwich WA (ed) Blochemistry of the Developing Brain, Vol 4. Marcel Dekker, Inc, New York, pp 143-186

5. Shah SN, Peterson NA, McKean CM 1972 Lipid composition of human cerebral white matter and myelin in PKU. J Neurochem 19:2369-2376

6. Curtius $\mathrm{H}-\mathrm{CH}$, Niederwieser A, Viscontini $M$, Leimbacher $W$, Wegman $H$, Blehova B, Rey F, Schaub J, Schmidt H 1981 Serotonin and dopamine synthesis in phenylketonuria. Exp Med Biol 133:277-291

7. McKean CM 1972 The effects of high phenylalanine concentrations on serotonin and catecholamine metabolism in the human brain. Brain Res 469 476

8. Krause W, Halminiski M, McDonald L, Dembure P, Salvo R, Freides D, Elsas L 1985 Biochemical and neuropsychological effects of elevated plasma phenylalanine in patients with treated phenylketonuria. J Clin Invest 75:4048

9. Monti JM 1982 Minireview, catecholamines and the sleep-wake cycle. Life Sci 122:1145-1157

10. Holtzman NA, Kronmal RA, Van Doornick W, Azen C, Koch R 1986 Effect of age at loss of dietary control on intellectual performance and behavior of children with phenylketonuria. N Engl J Med 314:593-598

11. Rolle-Daya H, Pueschel SM, Lombroso CT 1975 Electroencephalographic findings in children with phenylketonuria. Am J Dis Child 129:896-900

12. Donker DNJ, Reits D, Van Sprang FJ, Van Leeuwen WS, Wadman SK 1979 Computer analysis of the EEG as an aid in the evaluation of dietetic treatment in phenylketonuria. Electroencephalogr Clin Neurophysiol 46:205-213

13. Di Giorgis GF, Antonozzi I, del Castello PG, Rosano M, Loizzo A 1983 EEG as a possible prognostic tool in phenylketonuria. Electroencephalogr Clin Neurophysiol 55:60-68

14. Udenfriend S 1967 The primary enzymatic defect in PKU and how it may influence the central nervous system. In: Anderson, JA, Swaiman KF, (eds) Phenylketonuria and Allied Diseases. US Department Health, Education and Welfare, Washington, DC, pp 1-7

15. Johnson GA, Gren JM, Kupiecki R 1978 Radioenzymatic assay of DOPA (3,4-dihdyroxyphenylalanine). Clin Chem 24:1927-1930

16. Griffin RF, Elsas LJ 1975 Classic phenylketonuria diagnosis through heterozygote detection. J Pediatr 86:572-577

17. Cooney GA, Epstein CMC 1984 EEG frequency analysis in metrizamide radiography. Neurology 34:1102-1105

18. Barlow JS 1979 Computerized clinical electroencephalography in perspective. IEEE Trans Biomed Eng 26:377-391

19. Neter J, Wasserman W, Kutner MH 1985 Applied Linear Statistical Models 2nd ed. RD Irwin, Inc, Homewood, IL, pp 81-82

20. Fois A, Rosenberg C, Gibbs FA 1955 The EEG in phenylpyruvic oliophrenia Electroencephalogr Clin Neurophysiol 7:569-572

21. Clayton BE, Moncrief AA, Pommpiglione G, Shepard J 1966 Biochemical and EEG studies in phenylketonuric children during phenylalanine tolerance tests. Arch Dis Child 41:267-272

22. Spehlman R 1981 Generalized asynchronous slow waves. In: EEG Primer. Elseviere North-Holland Biomedical Press, New York, pp 359-377

23. Epstein CM, Andriola MR 1983 Alterations of the background activity. In: Introduction to EEG and Evoked Potentials. J. B. Lippincott Co, Philadelphia, pp 79-118

24. Behbehani AW 1985 Termination of strict dietary therapy in phenylketonuria Neuropediatrics 16:92-97

25. Pardridge W 1985 Potential effects of the dipeptide sweetener aspartame on the brain. In: Wurtman RJ, Wurtman JJ (eds) Nutrition and the Brain, Vol 7. Raven Press, New York, pp 199-241

26. Visek WJ 1984 Chronic ingestion of aspartame in humans. In: Stegink LD, Filer LJ (eds) Aspartame, Physiology and Biochemistry. Marcel Dekker, Inc New York, pp 495-508

27. Wurtman RJ 1982 Nutrients that modify brain function. Sci Am 245:50-60

28. Wurtman RJ, Hefti F, Melamed E 1981 Precursor control of neurotransmitter synthesis. Pharmacol Rev 32:315-335

29. Nagatsu T 1973 Biochemistry of Catecholamines: The Biochemical Method, University Park Press, Baltimore, pp 30-31 